\title{
Production, preventive and corrective maintenance planning for manufacturing systems under imperfect repairs
}

\author{
Annie Francie Kouedeu미 Jean-Pierre Kenné1, Abdelmoula Mohafid ${ }^{2}$ \\ ${ }^{1}$ Mechanical Engineering Department, Laboratory of Integrated Production Technologies, University of \\ Quebec, \\ École de technologie supérieure, 1100, Notre Dame Street West, Montreal (Quebec), Canada, H3C 1K3 \\ ${ }^{2}$ Organization and Production Engineering Department, IUT de Nantes, Rue Christian Pauc - La Chantrerie, \\ P.O. Box 50609 - 44306 Nantes Cedex 03 - France
}

This paper deals with the joint analysis of the optimal production and maintenance planning problems for a manufacturing system subject to random failures and repairs. When a machine fails down, an imperfect repair is undertaken. The objective of this study is to minimize a discounted overall cost consisting of preventive and corrective maintenance costs, inventory holding cost and backlog cost. A two-level hierarchical decision making approach, based on the determination of the mean time between failures (first level) and the statement of a joint optimization of production, preventive and corrective maintenance policies (second level) is proposed. Hence the production, preventive and corrective maintenance rates are determined in level 2 given the failure rates obtained from level 1. In the proposed model, the failure rate of the machine depends on the number of failures; hence, the control policies of the considered planning problem depend on the number of failures. A numerical example and a sensitivity analysis will illustrate the structure of the optimal control policies and the usefulness on the proposed approach.

Keywords: Imperfect repairs; Manufacturing Systems; Preventive and Corrective Maintenance.

\section{Introduction}

The reliability of a manufacturing system depends on the quality of its conception and the actions of the maintenance which are undertaken during its exploitation (production activities). This paper deals with the control problem of a stochastic manufacturing system consisting of one machine producing one part type. The stochastic nature of the system is due to the fact that the machine is subject to random breakdowns and repairs. Upon a failure of a component of the machine, an imperfect repair is undertaken. The machine dynamics is assumed herein to be described by a finite state semi-Markov chain. The decision variables are the production, the preventive and the corrective maintenance rates, which influence the availability of the system and the stock level. Many authors have contributed to the production planning problem of manufacturing systems (Boukas (1998)), without considering the failure rates depending on the number of failures and the production control in the same model. The aim of this paper is to propose a production control and maintenance (preventive and corrective) planning for a manufacturing system subject to imperfect repairs, when the failure rate increases with the number of failures. The proposed hierarchical approach consists in developing a model where at the first level; the parameters of the machine failure stochastic process are derived for each number of failures. At the second level, we determine the optimal production, preventive and corrective maintenance policies for a system that deteriorates with the number of failures. We obtain a 
production plan for the system, which minimizes inventory, backlog, preventive and corrective maintenance costs over an infinite planning horizon.

\section{Problem statement}

The considered manufacturing system consists of a single machine which can produce one-part type and its mode can be classified as operational, under repair, and under preventive maintenance. The dynamics of the machine is described by a continuous time semi-Markov process, with state or control dependent transition rates from one mode of the machine to another. The considered machine contains several components. The failure of a component causes the failure of the system. The replacement or the repair of the failed component puts the system in an operational mode. Note that the kind of repairable systems concerned in this paper are complex and consist of several components or subsystems. The failure of a component causes the system failure and replacing or repairing the faulty component lead the system in operating mode. The failure rate is deterministic and results from all interactions between the unities constituting the system. In order to increase the system capacity, we control the transition rate from operational mode to preventive maintenance mode and from corrective maintenance mode to operational mode. Hence, the transition matrix depends on the preventive and corrective maintenance rates. The system behavior is described by a hybrid state comprising both a discrete (state of the machine) and a continuous (stock level) component. When the machine is operational, it produces parts and when it is under preventive and corrective maintenance, it does not produce anything. The surplus may take either a positive value, called an inventory, or a negative value, called a backlog. Our objective is to control the production rate, the preventive and the corrective maintenance rates so as to minimize an expected discounted cost including inventory, backlog, preventive and corrective maintenance costs.

\section{Expected results}

The study of the production rate of the machine, in its operational mode shows that the computational domain is divided into three regions where the optimal production control policy consists of one of the following rules: (i) set the production rate of the machine to its maximal value when the current stock level is under a number of failure-dependent threshold value; (ii) set the production rate of the machine to the demand rate when the current stock level is equal to a number of failure-dependent threshold value; (iii) set the production rate of the machine to zero when the current stock level is larger than a number of failure-dependent threshold value. The control policy obtained is an extension to the so-called hedging point policy described in Akella and Kumar (1986). The preventive and corrective maintenance policies divide the computational domain into two regions where the preventive maintenance rate is set to zero and to its maximal value for backlog situation (or for uncomfortable stock levels) and for large stock levels respectively (Kenné and Nkeungoue (2008)). The corrective maintenance rate is set to its maximal and minimal values for backlog situation and for large stock levels respectively (Boukas (1998)). The optimal preventive and corrective maintenance policies, like the production policy, have a bang bang structure. By combining failure rate depending on the number of failures, preventive and corrective maintenance actions in production, the optimal threshold and the other parameters of the control policies increase when the number of failures increases. These results illustrate the contribution of the proposed model.

\section{Conclusion}


A hierarchical decision making approach in production, preventive and corrective maintenance planning with imperfect repairs problems under uncertainties has been proposed. We developed the stochastic optimization model of the considered problem with three decision variables (production rate, preventive and corrective maintenance rates) and one state variable (stock level). By controlling both production and maintenance rates, we obtained a near optimal control policy of the system through the implementation of the policy improvement algorithm (numerical methods). We have shown that the number of parts to hold in inventory increases when the number of breakdowns increases.

\section{References}

Akella, R. and Kumar P.R., 1986. «Optimal control of production rate in a failure prone manufacturing system», IEEE Trans. on Aut. Control, AC-31, 116-26.

Boukas, E. K., 1998. «Hedging point policy improvement», Journal of Optimization Theory and Applications, 97, 47-70

Kenné, J. P. and Nkeungoue N. L, 2008. « Simultaneous control of production, preventive and corrective maintenance rates of a failure-prone manufacturing system ». Applied Numerical Mathematics, 58(2),180-194. 